\title{
Revisiting the Field Synergy Principle in Elliptic Laminar Flow
}

\author{
Rawad Himo, Charbel Habchi \\ Notre Dame University - Louaize, Thermo-Fluids Research Group \\ P.O. Box: 72 Zouk Mikael, Zouk Mosbeh, Lebanon \\ rshimo@ndu.edu.lb; charbel.habchi@ndu.edu.lb
}

\begin{abstract}
Heat transfer enhancement is important in many engineering applications such as heat exchangers and chemical reactors. This enhancement could be boosted by locally analyzing the flow structure and its effect on temperature distribution. Thus the perception and deep comprehension of the correlation between the flow field and heat transfer is fundamental for many engineering applications, as well as in natural phenomena like weather prediction. The field synergy principle, based on the variation of the angle between the velocity vector and temperature gradient to describe and enhance the heat transfer process, has been widely used in the open literature to analyze convective heat transfer processes and to optimize flow configurations. However, we show in the present paper that the synergy angle cannot be used alone to describe the convection heat transfer coefficient in elliptic flows. The thermal diffusion in the streamwise direction plays an important role and thus the variation of the synergy angle contradicts the variation in the heat transfer coefficient. For this aim numerical simulations are carried out for a flow pas a backward facing step in laminar flow regime.
\end{abstract}

Keywords: Field Synergy, Advection Transport, Numerical Simulation, CFD, Convection, Backward Facing Step.

\section{Introduction}

In 1998, Guo et al. [1] published an article based on a parabolic boundary layer flow where they suggested three possible ways to increase the heat transfer coefficient by tuning the angle between the velocity vector and temperature gradient. This angle is defined as synergy angle. Further studies suggest extending this concept to elliptic flows to account for various heat transfer problems in engineering [2]. The compendium of these concepts is denoted the field synergy principle. However, the effect of the additional diffusive term in the flow direction $k\left(\partial^{2} T / \partial x^{2}\right)$ was neglected, Tao et al. [2] then claim that the field synergy principle works for both parabolic and elliptical flows.

Tao et al. [3] later compare the average synergy angle for cases with and without flow interruptions to bolster their concept. However, the effects of the local changes in the magnitudes of the velocity and temperature gradients were not considered. Not to mention, the absence of the exclusion of the synergy angles outside the thermal boundary layers in the calculation of the overall synergy angle. Last but not least, the validation of the assumption of that the thermal diffusive transport is insignificant was not proved in elliptic flows. For instance, $\mathrm{Wu}$ and Tao $[4,5,6]$ performed studies on flows with longitudinal vortex generators without taking into consideration the local variation and comprehension of the field synergy principle.

In a similar manner, plain plate and triangular wavy fin-and-tube heat exchangers were analyzed numerically by He $e t$ al. [7] and Cheng et al. [8], respectively, by considering always global variation of the field synergy angle versus the overall Nusselt number.

Meng et al. [9] numerically optimized multi-longitudinal vortices flow in tube based on what is referred to as the synergy equation, without showing the effect of the synergy angle and its contribution to the enhancement of the heat transfer.

The work done by Ma et al. [10] on the synergy field vis-à-vis efficiency evaluation is best discussed in Bejan's famous Heatlines (1983) vs Synergy (1998) [11] paper where he mentions the flaws in this principle. Bejan [11] pointed the opposite behavior of the synergy angles $\theta$ and $\beta$ defined by Ma et al. [10], where $\beta$ behaves inversely with the efficiency evaluation criterion, hence in opposition to the whole field synergy principle.

In this paper, we revisit the field synergy principle in elliptic flows where we highlight the importance of the diffusive term in the heat transfer phenomena which was previously neglected in the field synergy principle. Moreover, we show 
that the field synergy angle alone cannot describe the behavior of the heat transfer coefficient. This is done by analyzing the local variation of the different components of the energy equation for a laminar flow past a backward facing step.

\section{Numerical Procedure}

\subsection{Computational Domain}

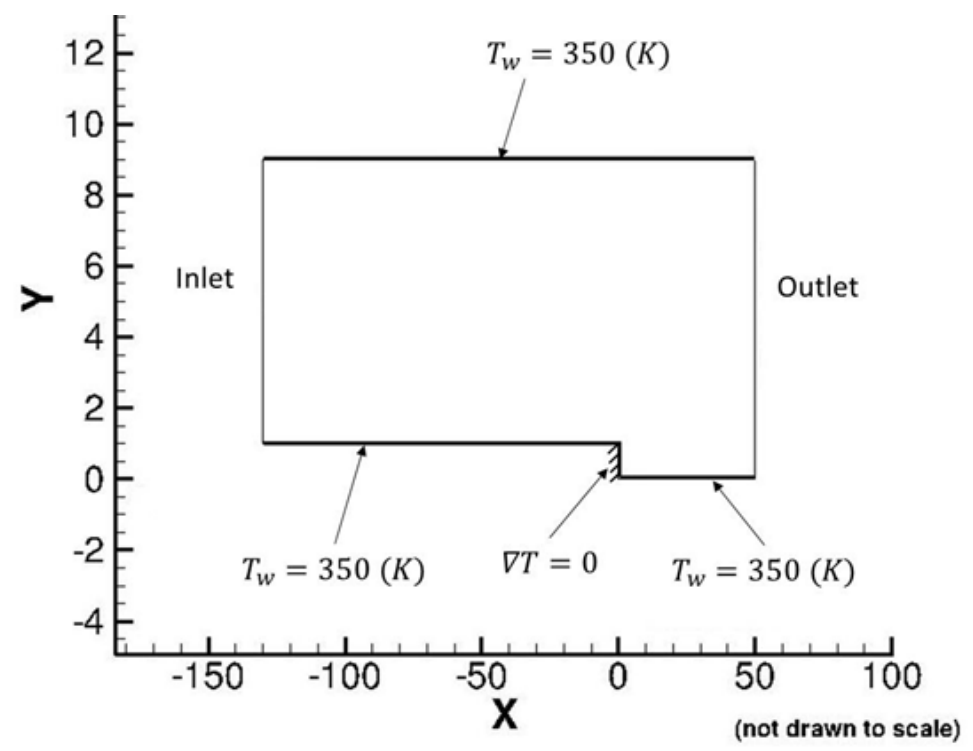

Fig. 1: Computational domain and boundary conditions.

To consistently test the validity of this field synergy principle and the assumption that the diffusive transport in the flow direction is insignificant in elliptical flows, the wall heat flux must only emanate vertically (keeping in mind that heat flux is normal to the boundary). Consequently, all horizontal walls are set to a constant temperature $T_{w}=350 \mathrm{~K}$ whereas the only vertical wall, namely the backward facing step, is thermally insulated.

The working fluid is air, entering at the inlet with a uniform velocity profile at constant temperature $T_{w}=300 \mathrm{~K}$. Atmospheric pressure is prescribed at the domain outlet. No-slip boundary conditions are prescribed on all solid surfaces.

The flow Reynolds number is based on the step height $H=1 \mathrm{~m}$ and simulations are performed for Reynolds numbers $R e_{H}=36$ and $R e_{H}=36,000$. The higher Reynolds number is used to validate our numerical simulations by using the $k-\omega$ SST turbulence model while the lowest Reynolds is used for the physical analysis in laminar flow.

\subsection{Governing Equations and Numerical Method}

The laminar flow field is governed by the two-dimensional (2D) Navier Stokes equations. The continuity and momentum equations for an incompressible Newtonian fluid are as follows:

$$
\begin{gathered}
\nabla \cdot \mathbf{u}=0 \\
\mathbf{u} \cdot \nabla \mathbf{u}=-\frac{1}{\rho} \nabla p+\frac{1}{\rho} \nabla \cdot \mathbf{S}
\end{gathered}
$$

where $\mathbf{S}$ is the viscous stress tensor.

The energy equation is also computed in the fluid domain and is given by:

$$
\rho c_{p} \nabla \cdot(\mathbf{u} T)=\nabla \cdot(k \nabla T)
$$


where $c_{p}$ is the specific heat and $k$ is the molecular thermal conductivity.

The solver used the CFD code ANSYS Fluent 18.0 [13], which is based on cell-centered finite volume discretization method. The governing equations are solved sequentially with double precision and a second-order upwind scheme [15] for spatial discretization of the convective terms. The diffusion terms are central differenced and second-order accurate. Pressure-velocity coupling is achieved by the SIMPLE algorithm [13].The residual value $10^{-6}$ is set as the convergence criterion for the solutions of the flow and energy equations.

\section{Results and Discussion}

\subsection{Meshing and Numerical Validation}

A non-uniform structured three-dimensional mesh is generated while paying special attention to the near-wall refinement at all solid boundaries, so as to take into account the high velocity and temperature gradients in these regions. To determine the appropriate mesh density for solution grid independence, the solver is run with increasing mesh densities until no significant effect on the results is detected. The mesh validity verification is performed by using the method proposed by Celik [16] where the grid convergence index (GCI) and the apparent order of convergence $p_{c}$ can be obtained. The mesh validity verification is applied to the global Nusselt number $\mathrm{Nu}$ :

$$
N u=\frac{H h}{k}
$$

where $h$ is the overall convective heat transfer coefficient $\left(W / m^{2} K\right), H$ the step height, and $k$ is the thermal conductivity of the working fluid.

In Eq. $4, h$ is calculated from the logarithmic mean temperature difference:

$$
h=\frac{\dot{q}}{A \Delta \mathrm{T}_{\mathrm{lm}}}
$$

where $\dot{q}$ is the overall rate of heat transfer:

$$
\dot{q}=\dot{m} c_{p} \Delta T
$$

where $\dot{m}$ is the mass flow rate $(\mathrm{kg} / \mathrm{s})$ and $\Delta T$ is the bulk temperature difference between the inlet and outlet:

$$
\Delta T_{l m}=\frac{\left(T_{w}-T_{\text {in }}\right)-\left(T_{w}-T_{\text {out }}\right)}{\ln \left(\frac{T_{w}-T_{\text {in }}}{T_{w}-T_{\text {out }}}\right)}
$$

The uncertainty in the fine-grid solution is found to be about $0.02 \%$ and the order of convergence is 7.6. For more details about the calculation of the parameter GCI and $p_{c}$, the readers can refer to Celik [16]. It should be noted that the mesh study presented here is performed for the highest Reynolds number i.e. $R e_{H}=36,000$ representing the critical case due to highest fluctuations and gradients. The numerical simulations are performed on eight parallel Intel Core i7-7700 $2.80 \mathrm{GHz}$ processors. Each run takes less than one day.

The numerical results are validated with NASA's experimental data [12] for the local friction coefficient as shown in Fig.2. From this figure fair agreement is observed between the present numerical result and that obtained from experimental study. 


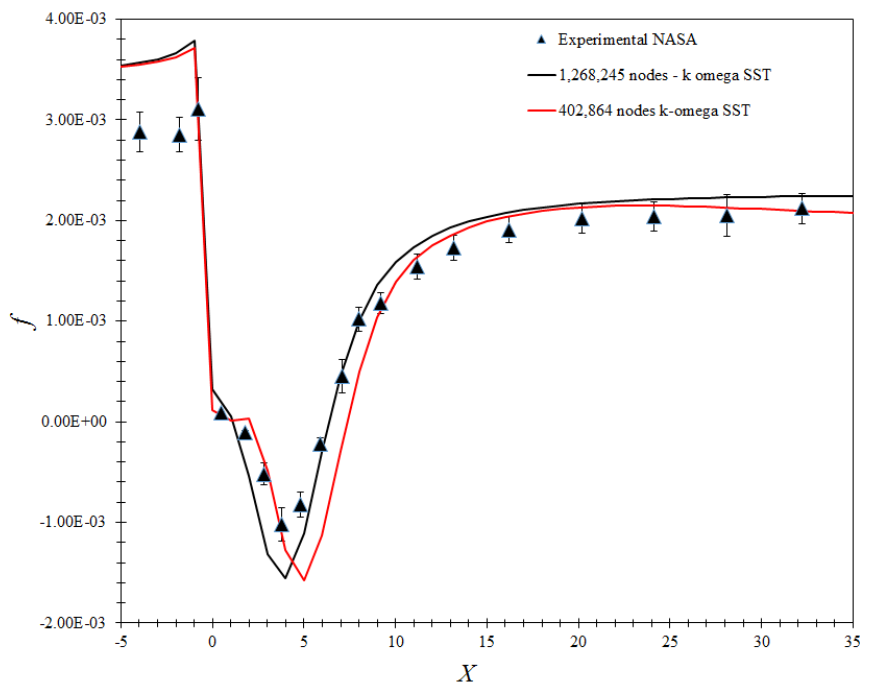

Fig. 2: Numerical validation with NASA’s experimental data.

\subsection{Examining the Field Synergy Principle}

Guo's [1] "Novel concept for convective heat transfer" suggest increasing the convective heat transfer by changing the synergy angle. They overlooked the fact that the simplified version of the energy equation becomes obsolete when the synergy angle is changed, since the diffusive transport in the horizontal direction can no longer be neglected because the whole structure of the flow has changed locally.

A more correct representation of the heat transfer for a horizontal heating in an elliptical flow should be as follows:

$$
\rho c_{\mathrm{p}}\left(u \frac{\partial T}{\partial x}+v \frac{\partial T}{\partial y}\right)=\frac{\partial}{\partial x}\left(k \frac{\partial T}{\partial x}\right)+\frac{\partial}{\partial y}\left(k \frac{\partial T}{\partial y}\right)
$$

Isolating and integrating in the $y$ direction:

$$
\mathrm{q}_{\mathrm{w}}=k \frac{\partial T}{\partial y}=\int_{0}^{\delta_{t h}} \rho \mathrm{c}_{\mathrm{p}}\left(u \frac{\partial T}{\partial x}+v \frac{\partial T}{\partial y}\right)-\int_{0}^{\delta_{t h}} \frac{\partial}{\partial x}\left(k \frac{\partial T}{\partial x}\right)
$$

Calculating the local Nusselt number based on the step height $H$ from $q_{w}$, the validity of the Field Synergy principle can be put to the test. The results of the local Nusselt are shown in Fig. 3 comparing Nu obtained from the wall heat flux defined in Eq. 6 to that obtained from Eq. 9. In addition, the synergy angle is plotted on the secondary vertical axis to juxtapose its effect on the span-average local Nusselt number.

The Field Synergy principle's assumption is valid throughout the entrance and the developed region of the flow, except near the proximity of the step as shown in Fig. 3 (a). That's where the synergy angle is varied greatly, hence the claim that the convective heat transfer can be improved by increasing the synergy angle becomes unreliable since the equation defining this principle is not fully representative of the heat transfer. A close-up on the variation of the Nusselt numbers near the step is shown in Fig.3 (b), where it is clear that the behaviour of the transport described in the Field Synergy Principle $[1,2]$ is completely different than the actual phenomenon. However, the present study's claim of the importance of the diffusive transport in the $\mathrm{x}$ direction upon the change in the flow structure is clearly representing the transfer of the thermal energy. 


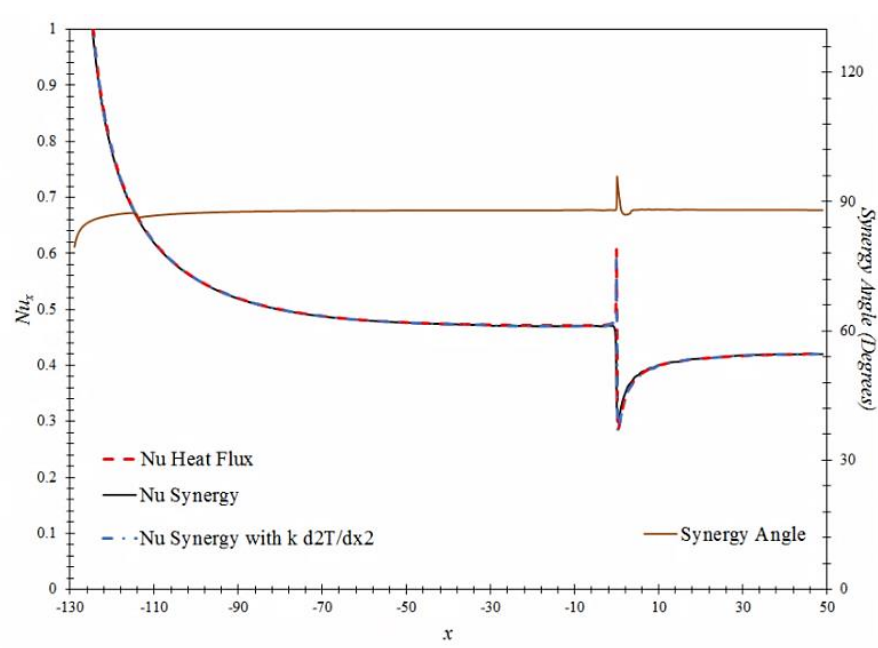

(a)

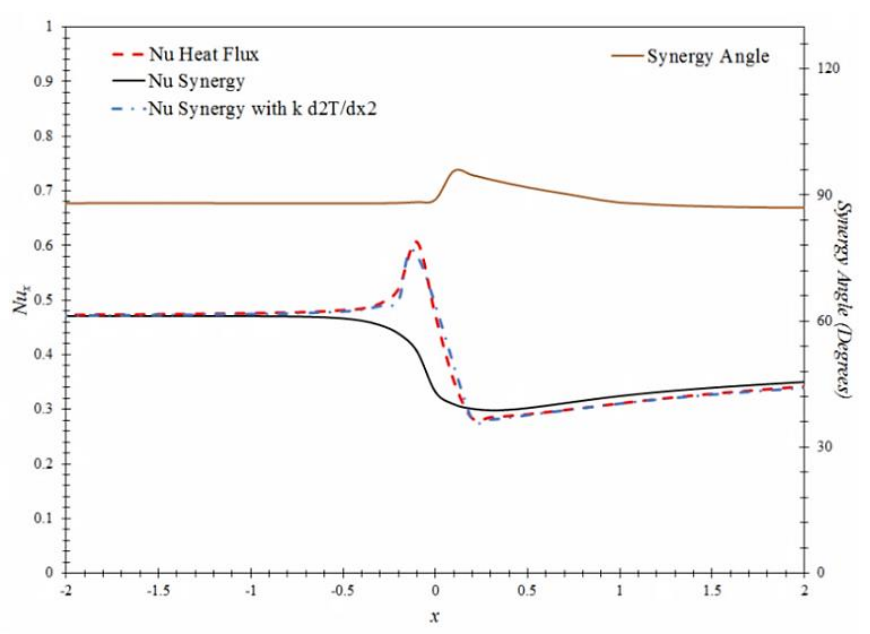

(b)

Fig. 3: Local nusselt number for the different methods and the synergy angle (a) for the whole domain and (b) close-up on

$$
-2<x<2 \text {. }
$$

Obviously, advection is not solely responsible for the heat transfer in this elliptic flow. Especially when the synergy angle is changing, which further supports the claim in this manuscript. This issue will be further elaborated by showing the contribution of the advective $\psi$ and the diffusive $\xi$ terms defined respectively in the following expressions:

$$
\begin{gathered}
\psi=\int_{0}^{\delta_{t h}} \rho \mathrm{c}_{\mathrm{p}}\left(u \frac{\partial T}{\partial x}+v \frac{\partial T}{\partial y}\right) \\
\xi=\int_{0}^{\delta_{t h}} \frac{\partial}{\partial x}\left(k \frac{\partial T}{\partial x}\right)
\end{gathered}
$$

It can be noticed from Fig. 4 that near the step region where the synergy angle exhibits changes, the contribution of diffusive term cannot be neglected because its magnitude reaches values higher than advection. This results in the contrast between the heat transfer coefficient and the field synergy angle.

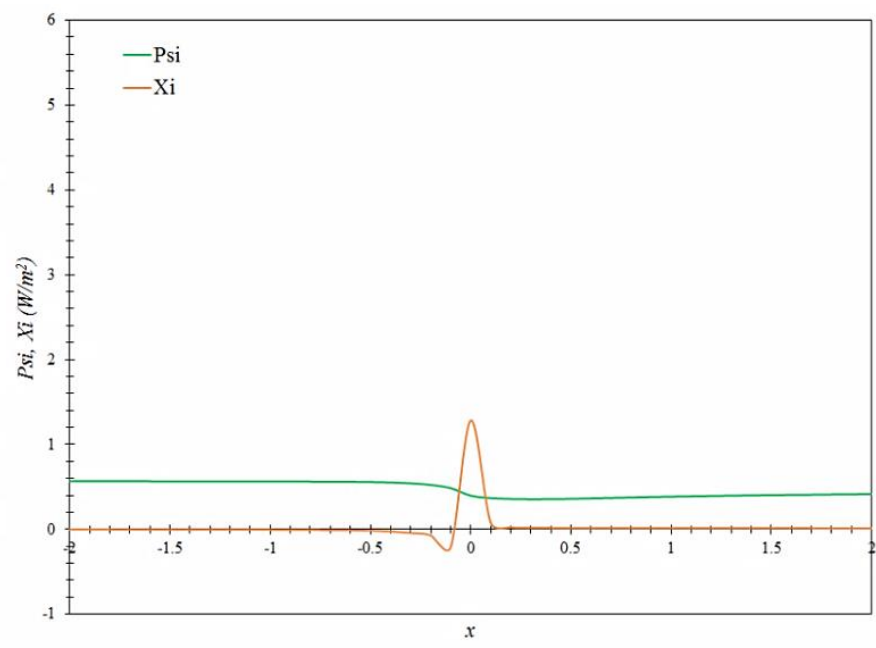

Fig. 4: Close up on the variation of $\psi$ and $\xi$ terms with the streamwise direction. 


\section{Conclusion}

Heat transfer enhancement is fundamental issue in many engineering applications such as heat exchangers, chemical reactors, internal combustion engines and plasma physics like in tokomaks. This enhancement could be increased by locally analyzing the flow structure and its effect on temperature distribution. Thus the comprehension of the correlation between the flow field and heat transfer is fundamental for many engineering applications, as well as in natural phenomena like weather prediction.

The field synergy principle, based on the variation of the angle between the velocity vector and temperature gradient to describe and enhance the heat transfer process, has been widely used in the open literature to analyze convective heat transfer processes and to optimize flow configurations.

In this paper, we revisit the field synergy principle in elliptic flows where we highlight the importance of the diffusive term in the heat transfer phenomena which was previously neglected in the field synergy principle. Moreover, we show that the field synergy angle alone cannot describe the behavior of the heat transfer coefficient. This is done by analyzing the local variation of the different components of the energy equation for a laminar flow past a backward facing step.

The numerical results are compared and validated against experimental data obtained in the open literature.

\section{References}

[1] Z.-Y. Guo, D.-Y. Li, B.-X. Wang, "A novel concept for convective heat transfer enhancement," Int. J. Heat Mass Transfer, vol. 41, pp. 2221-2225, 1998.

[2] W.-Q. Tao, Z.-Y. Guo, B.-X. Wang, "Field synergy principle for enhancing convective heat transfer its extension and numerical verifications," Int. J. Heat Mass Transfer, vol. 45 pp. 3849-3856, 2002.

[3] W.-Q. Tao, Y.-L. He, Q.-W. Wang, Z.-G. Qu, F.-Q. Song, "A unified analysis on enhancing single phase convective heat transfer with field synergy principle," Int. J. Heat Mass Transfer, vol. 45, pp. 4871-4879, 2002.

[4] J.-M. Wu, W.-Q. Tao, "Numerical study on laminar convection heat transfer in a rectangular channel with longitudinal vortex generator. part a: Verification of field synergy principle," Int. J. Heat Mass Transfer, vol. 51, pp. 1179-1191, 2008.

[5] J.-M. Wu, W.-Q. Tao, "Numerical study on laminar convection heat transfer in a channel with longi- tudinal vortex generator. part b: Parametric study of major influence factors," Int. J. Heat Mass Transfer, vol. 51, pp. 3683-3692, 2008.

[6] J.-M. Wu, W.-Q. Tao, "Investigation on laminar convection heat transfer in fin-and-tube heat exchanger in aligned arrangement with longitudinal vortex generator from the viewpoint of field synergy principle," App. Thermal Eng., vol. 27, pp. 2609-2617, 2007.

[7] Y.-L. He, W. Tao, F. Song, W. Zhang, "Three-dimensional numerical study of heat transfer characteristics of plain plate fin-and-tube heat exchangers from view point of field synergy principle," Int. J. Heat Fluid Flow, vol. 26, pp. 459-473, 2005.

[8] Y. Cheng, T. Lee, H. Low, Numerical analysis of periodically developed fluid flow and heat transfer characteristics in the triangular wavy fin-and-tube heat exchanger based on field synergy, Numerical Heat Transfer, Part A, vol.53, pp. 821-842, 2008.

[9] J.-A. Meng, X.-G. Liang, Z.-X. Li, "Field synergy optimization and enhanced heat transfer by multi- longitudinal vortexes flow in tube," Int. J. Heat Mass Transfer, vol. 41, pp. 3331-3337, 2005.

[10] L. Ma, J. Yang, W. Liu, X. Zhang, "Physical quantity synergy analysis and efficiency evaluation criterion of heat transfer enhancement," Int. J. Thermal Sciences, vol. 80, pp. 23-32, 2014.

[11] A. Bejan, "Heatlines (1983) versus synergy (1998)," Int. J. Heat Mass Transfer, vol. 81, pp. 654-658, 2015.

[12] C. Rumsey, (2016). Langley Research Center, National Aeronautics and Space Administration (NASA) 2d backward facing step. [Online]. Available: https://turbmodels.larc.nasa.gov/backstep_val.html

[13] ANSYS, Academic Research, Release 18.0.

[14] F. Menter, "Two-equation eddy-viscosity turbulence models for engineering applications," AIAA J., vol. 32, pp. 1598-1605, 1994.

[15] R. Warming, R. Beam, "Upwind second-order difference schemes and applications in aerodynamic flows," AIAA J., vol. 14, pp. 1241-1249. 1976. 
[16] I. Celik, U. Ghia, P. Roache, H. Freitas, Procedure for estimation and reporting of uncertainty due to discretization in CFD applications, J. Fluids Eng., vol.130, 078001, 2008. 